\title{
Start small, think big
}

\author{
Jonathan M. Chen, MD \\ From the Division of Congenital Cardiac Surgery, Department of Surgery, University of Washington School of \\ Medicine, Seattle, Wash. \\ Disclosures: Author has nothing to disclose with regard to commercial support. \\ Received for publication May 24, 2017; accepted for publication May 31, 2017; available ahead of print June 29, \\ 2017. \\ Address for reprints: Jonathan M. Chen, MD, Seattle Children's Hospital, 4800 Sand Point Way NE - RC 2.820, \\ Seattle, WA 98105 (E-mail: jmchen@uw.edu). \\ J Thorac Cardiovasc Surg 2017;154:1984-5 \\ $0022-5223 / \$ 36.00$ \\ Copyright $(5) 2017$ by The American Association for Thoracic Surgery \\ http://dx.doi.org/10.1016/j.jtcvs.2017.05.093
}

\section{“It's not an experiment if you know it's going to work."}

$$
\text { -Jeff Bezos }
$$

The development of a novel means to mitigate the prothrombotic and proinflammatory insurrection generated by cardiopulmonary bypass $(\mathrm{CPB})$ remains one of the fundamental holy grails of cardiac surgery. In this setting, acute lung injury (ALI) often represents the common terminus of several overlapping cascades, which at its extreme can lead to acute respiratory distress syndrome and significant morbidity and mortality. In this issue of the Journal, Hirao and colleagues ${ }^{1}$ report the attenuation of ALI and apoptosis in a rat CPB model based on preoperative administration of recombinant human soluble thrombomodulin (rTM), a thrombin-binding anticoagulant cofactor demonstrated to have a similar salutary effect in the setting of disseminated intravascular coagulation and sepsis in humans. ${ }^{1,2}$

The authors comprehensively demonstrate their findings across several investigational domains-physiologic variables, cytokine and HMGB1 levels, histology, immunohistochemistry, terminal deoxynucleotidyl transferase dUTP nick-end labeling assay, caspase-3 immunostaining, and cleaved content - thereby supporting their speculation that rTM exerted its favorable effect by reducing the overall systemic inflammatory cytokine release and local lung inflammation (or apoptosis), thereby limiting ALI both during and up to 4 hours after CPB. These findings are in many ways a logical extension of previous experimental studies in vivo and in vitro first suggesting that rTM may have considerable effect in mitigating the ALI associated with various systemic inflammatory diseases. ${ }^{3}$

However, although precise in its implementation (and I entreat the reader to watch the video of the authors' technique associated with the article), the rat CPB model will require considerable modifications before it can be declared to simulate clinical cardiac surgery accurately. ${ }^{1}$ Many important common aspects of standard open-heart procedures that could considerably alter the benefit of rTMdiffering degrees of hemodilution, cardiotomy suction/

\section{References} 2013;41:2069-79.

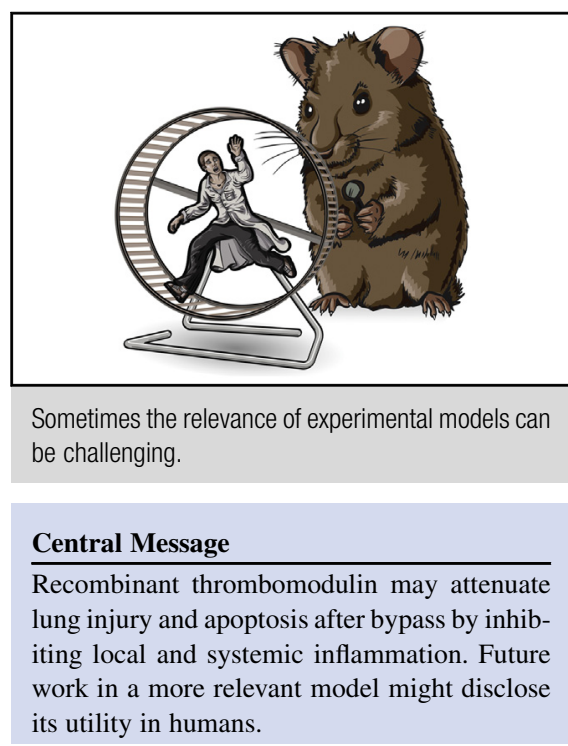

See Article page 1973.

reservoir, administration of cardioplegia, centrifugal pumps, blood transfusion-in addition to the more fundamental question of how closely the rodent and human inflammatory systems resemble one another, all argue in favor of ongoing analysis of this novel agent in an experimental animal model with better fidelity to the human condition. Only then can appropriate dosing be assessed (with particular attention to the potential negative antithrombotic side effect of rTM) and can subjects be followed beyond a few hours postoperatively. ${ }^{4}$

Nevertheless, Hirao and colleagues have shown through a series of elegant analyses the proof of concept, namely that "upstream" modulation of inflammation can have considerable downstream benefit in mitigating injury and apoptosis. The next step in this trajectory will be the move from small to big-both in experimental model and overall concept - so that the vision of a reduction in ALI and acute respiratory distress syndrome after cardiac surgery might become reality; the result could make us all breathe more easily.

1. Hirao S, Minakata K, Masumoto H, Yamazaki K, Ikeda T, Minatoya K, et al. Recombinant human soluble thrombomodulin prevents acute lung injury in a rat cardiopulmonary bypass model. J Thorac Cardiovasc Surg. 2017;154:1973-83.e1.

2. Vincent J-L, Rames MK, Ernest D, LaRosa SP, Pachl J, Aikawa N, et al. A randomized, double-blind, placebo-controlled, Phase $2 \mathrm{~b}$ study to evaluate the safety and efficacy of recombinant human soluble thrombomodulin, ART-123, in patients with sepsis and suspected disseminated intravascular coagulation. Crit Care Med. 
3. Hagiwara S, Iwasaka H, Matsumoto S, Hasegawa A, Yasuda N, Noguchi T. In vivo and in vitro effects of the anticoagulant, thrombomodulin, on the inflammatory response in rodent models. Shock. 2010;30:282-8.
4. Gomi K, Zushi M, Honda G, Kawahara S, Matsuzaki O, Kanabayashi T, et al. Antithrombotic effect of recombinant human thrombomodulin on thrombin-induced thromboembolism in mice. Blood. 1990;75:1396-9. 